\title{
Estrategias efectivas para aumentar la cobertura del Papanicolaou en el programa de detección del cáncer cervicouterino
}

PAOLA SALAS(1), SERGIO GUZMAN(1), GISELLE CAZOR(2)

\section{RESUMEN}

El aumento de las coberturas del Papanicolaou depende de múltiples factores donde se destaca la motivación de los equipos de salud, en especial de la profesional matrona, asi como de una red de apoyo interdisciplinario y comunitaria destinada a captar mujeres, y el concepto de auto-cuidado que tenga internalizado cada mujer. El objetivo de este trabajo es dar a conocer estrategias de salud publica que permitieron aumentar la cobertura del Papanicolaon (Pap) vigente en mujeres beneficiarias en $u n 12$ y $8 \%$ en los Servicios de Salud Valdivia y Metropolitano Norte. Las estrategias de salud publica probadas en este estudio como efectivas fireron: a) fomento de la informática con: la instalación del cito-expert en la atención primaria, planilla de cálculo de coberturas y estadistica mensual, planilla de contra-referencia de pacientes atendidas en la UPC y envio via e-mail cada tres dias; b) Captación de monitoras de Pap en la comnidad; c) Investigación operativa y capacitación permanente a matronas, médicos y monitoras de Pap. d) Implementación de un banco de proyectos de promoción. Esto traducido en el efecto en la salud de la mujer ha significado diagnosticar NIE III cuatro veces más que en 1993, detectar el cáncer invasor en etapas iniciales (etapas I y II), y descender consecuentemente la mortalidad por esta enfermedad, alcanzando actualmente a una tasa de 4,6 por 100.000 mujeres mayores de 15 años en el Servicio de Salud de Valdivia.

Palabras claves: neoplasmas del cuello uterino, salud publica, estrategias locales.

\begin{abstract}
EFFECTIVE STRATEGIES TO INCREASE THE PAPANICOLAU COVERAGE IN THE CERVICOUTERINE CANCER DETECTION PROGRAM

The increase in Papanicolan coverage depends on multiple factors, in which the motivation of health teams stands ont, specially that of the professional midwife, aided by an interdisciplinary and community support network destined to atract women, and the concept of self care that each woman has internalized. The objective of this work is that of making available public health strategies allowing to bring about an increase in Papanicolan (PAP) coverage in beneficiary women by a $12 \%$ and $8 \%$ in the Valdivia and North Metropolitan Health Services respectively. The public health strategies proved as effective in this study were: a) promotion of the informative aspect, with installation of the cito-expert in primary attention, statements of coverage calculations
\end{abstract}

(1) Servicio Salud Valdivia psalas asaludvald cl

(3) Servicio de Salud Metropolitano Norte. 
and monthly statistics, counter-reference statement of patients attended at the Cervical Pathology Unit (UPC) and e-mail dispatch every three days; b) winning over of Pap monitors in the community; c) operative research and permanent training of midwives, physicians and Pap monitors; d) implementation of a promotion projects bank. This, translated into the effect on women's health, has implied diagnosing intraepithelial neoplasia III (NIE III) four times as much as in 1993 and invasive cancer in its intial stages (stages I and II) and, consequently, decreasing mortality from this disease which, at present, reaches a rate of 4.6 per 100.000 women over 15 years of age in the Valdivia Health Service.

Key words: uterine neck neoplasms; public health; local strategies.

\section{INTRODUCCIÓN}

El cáncer cervicouterino es un problema prevalente en las mujeres de todo el mundo, alcanzando a 500.000 muertes anuales!. El principal impacto se ha logrado en países con programas de pesquisa precoz bien implementados y que han obtenido coberturas del Pap sobre el $80 \%$, permitiendo evidenciar la disminución en la incidencia y mortalidad por esta causa ${ }^{2,3}$. En Chile, a pesar de estar implementado el programa de detección precoz por cáncer cervicouterino, la cobertura del Pap vigente en las beneficiarias para el grupo de 25-64 años, no supera el $66 \%$ en el año $2003 \mathrm{y}$ la mortalidad por cáncer en la mujer, ubica al cáncer cervicouterino en el cuarto lugar con una tasa de 9,6 por 100.000 (900 muertes), sólo por debajo del cáncer de vesícula y vía biliar, gástrico y mamas ${ }^{4}$. Por tal motivo, uno de los aspectos relevantes de la salud pública de nuestro país ha sido aumentar la cobertura del Pap como determinante en los cambios que se pueden lograr en la incidencia y mortalidad por esta causa.

Los registros en Chile revelan coberturas dificiles de precisar y discordantes según la fuente de información ${ }^{5}$. Por este motivo se buscó validar los registros, así como obtener aumentos reales y mantenidos de la cobertura que permita impactar en la salud de la mujer, realizando una citología efectiva y focalizada en grupos de riesgo. Se presenta a continuación la experiencia de los Servicio de Salud Valdivia y Metropolitano Norte.

\section{MATERIAL Y MÉTODO}

Para enunciar los resultados, se describirá el proceso para cada estrategia de salud pública desarrollada tendiente a aumentar en su conjunto la cobertura del Pap, donde se describen: la informática aplicada al programa de detección precoz (implementación de cito-expert en la red de atención primaria y unidad de patología cervical (UPC), planilla automática de cálculo de coberturas del Pap vigente y planilla de contra-referencia desde UPC a la atención primaria); incorporación de la comunidad al programa de detección precoz; capacitación e investigación operativa permanente, y la implementación de un banco de proyectos de promoción.

En los cálculos de cobertura de Pap vigente, se estudió la población femenina beneficiaria que se ha realizado el Pap en los últimos tres años según recomendaciones del Ministerio de Salud, siendo dicha población separada por grupos entre 25-64 años y, la de mayor riesgo, entre 35-64 años.

Para el estudio de la incidencia y mortalidad se utilizó como denominador al total de la población femenina mayor de 15 años según lo proyectado por el Instituto Nacional de Estadísticas cada año ${ }^{6}$, así como los casos nuevos diagnosticados histológicamente para el cáncer in sith (NIE III) e invasor y los certificados de defunción existente en la base de datos de los Servicio de Salud analizados.

\section{RESULTADOS}

Estrategia $N^{0}$ 1: Informática aplicada al programa de detección precoz.

a) Implementación de Cito-expert (Base de 
datos de PAP) en la red de atención primaria y unidad de patología cervical.

Teniendo como base que existe un sobreregistro de mujeres con Pap vigente y que los informes de coberturas mediante tarjeteros no reflejan muchas veces el estado real del Pap de cada mujer (algunas se realizan el examen citológico el mismo año en diferentes establecimientos), la búsqueda de datos confiables con la finalidad, además, de optimizar los registros y agilizar la búsqueda de pacientes nuevas y con Pap atrasados, se instaló el programa Cito-expert, (que es una base de datos de los Pap, alimentada a diario por cada unidad de anatomía patológica) en los 21 y 18 establecimientos de atención primaria y UPC del Servicio de Salud Valdivia y Metropolitano Norte, respectivamente.

El Cito-expert en el Servicio de Salud Metropolitano Norte fue instalado en la SOME de los establecimientos de salud, teniendo como usuarios a funcionarias de dicha unidad (Figura 1), los que daban hora para la toma de Pap con matrona cuando la usuaria estaba con su PAP atrasado o nunca se había realizado el examen. En el Servicio de Salud Valdivia, se capacitó a las matronas encargadas del programa de detección precoz en cada establecimiento de atención primaria, quienes realizaron la "limpieza de tarjeteros", que involucraba verificar el estado de los Pap del total de pacientes con tarjetas de seguimiento, es decir que alguna vez en su vida habian realizado un examen y separar las inasistentes al Pap en los últimos 3 años para su posterior citación.

Esta base de datos ofrece, además, como productos; nóminas de mujeres con Pap atrasados, mujeres por venir, focalización según grupo de riesgo, informe de calidad de toma de muestra por profesional, cobertura de Pap, etc. (Figura 2).

b) Planilla automática de cálculo de coberturas del Pap vigente.

Se elaboró una planilla de cálculo de cobertura automática en conjunto con el Departamento de Informática del Servicio de Salud Valdivia, que incluye el cálculo de coberturas del Pap vigente de mujeres cuyas edades se separan cada 5 años, donde las matronas digitan los casos ingresos (nuevos y atrasados) y los egresos a la toma de Pap (mujeres que no repiten Pap en la fecha indicada para permanecer en la cobertura, así como las que cambian domicilio, fallecen etc). Con el objeto de actualizar sus coberturas mensualmente, la planilla hace los cálculos y pone la población existente para el próximo mes, asi como la adición de casos para la evaluación final en diciembre de cada año. Esta información es remitida al final de cada mes vía e-mail o disquete a la UPC donde se imprime y se realiza un monitoreo por parte de

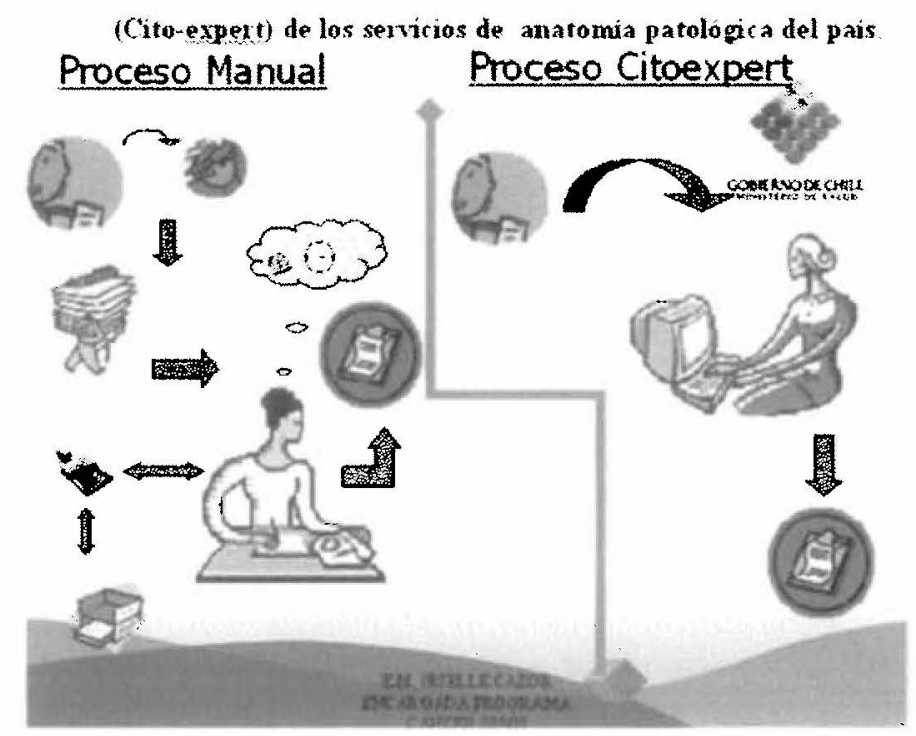

Figura 1. Proceso de búsqueda del estado de Papanicolaou de beneficiarias, registradas desde el año 1996 en base de datos (Cito-expert) de los servicios de anatomía patológica del país. 


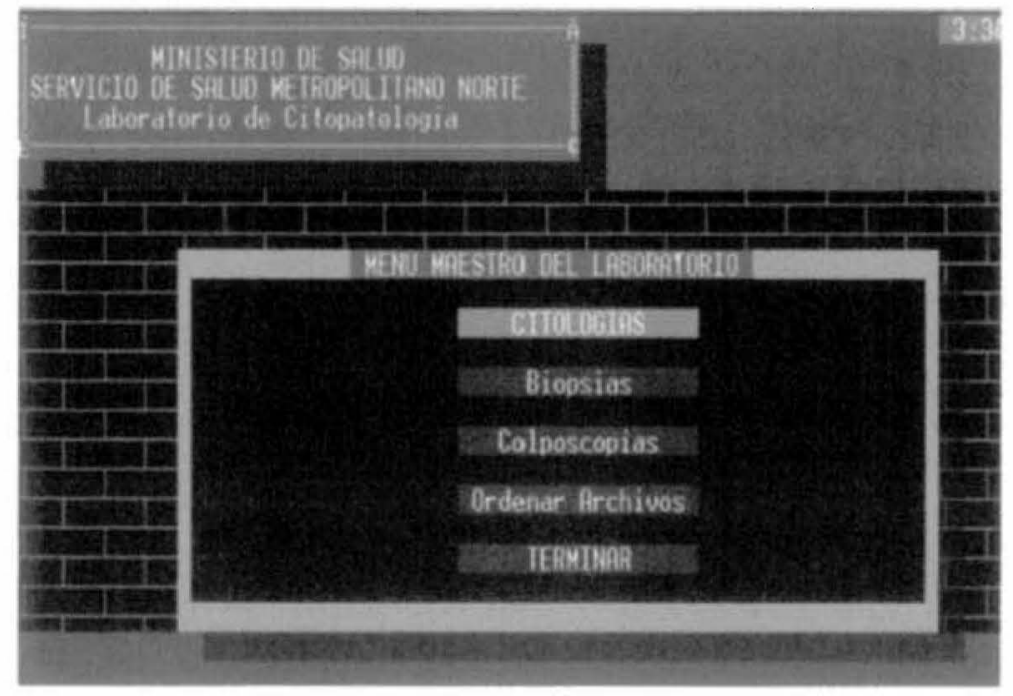

Figura 2. Programa computacional Cito-expert, en el menú de utilidades. un equipo coordinador del estado de avance o descenso de las coberturas en cada establecimiento de salud de la atención primaria. (Figura 3).

c) Planilla de contra-referencia desde Unidad de Patología Cervical a atención primaria:

Se creó una planilla de contra-referencia en el programa computacional Excel, donde se digita a diario: nombre, diagnóstico de derivación, evaluación en UPC, indicaciones y fecha de asistencia del total de pacientes evaluadas en
UPC. Esta actividad está a cargo de la matrona coordinadora y es remitida cada tres días a cada establecimiento de la atención primaria vía e-mail. Esta información, a su vez, es traspasada a las fichas clínicas de los establecimientos de origen por la matrona encargada en cada establecimiento de atención primaria, quedando la información sobre el estado actual de la usuaria que consulta al nivel secundario o UPC, disponible para el equipo de salud local (Figura 4).

\begin{tabular}{|c|c|c|c|c|c|}
\hline \multicolumn{4}{|l|}{ SERUTCIO OE SAL WO VALOUUA } & & \\
\hline BERTOQELAS PERSONAS & & & & & \\
\hline \multicolumn{6}{|c|}{ PROGRAAA DE CANCER CBRUICO GTERAHO } \\
\hline & & & & & \\
\hline \multicolumn{6}{|c|}{ OEE PAOLA SALAS MATBOMA UPC. } \\
\hline \multicolumn{6}{|c|}{ A: MATRONA ENGARGADA PROG.GA CUT } \\
\hline & & & & & \\
\hline \multicolumn{6}{|c|}{ 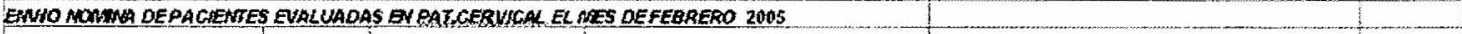 } \\
\hline & & & & & \\
\hline \multirow{2}{*}{ ESIABGLECNAENTO: } & & & ceV. & & \\
\hline & & 1. & & & \\
\hline MONTRE & RUT & DIAG DERAACKN & EVALUACONLPC & HOWCAGROAFS & EECHACOA \\
\hline$\times \times \times \times \times \times \times \times \times \times \times \times \times \times x$ & & PAPA & COLP CONOLOHA & SE DIFIERE TRATAATIENTO CONTROL EN $4 \mathrm{MESES}$ & 011022000 \\
\hline$x \times x \times \times \times \times 00 \times \times \times \times x$ & $71321726-1$ & MIE II TRATADA & COL F EXOC SANO & QL TA UPC PBP ANUAL & 02,121000 \\
\hline$x \times \times \times \times \times \times 0 \times x \times x) \times$ & $13117214-1$ & MIE III TRATADA & COLP EXOC SANO & ALTA UPC, PAP ANUAL & 10025000 \\
\hline XXXYYXXXXXXXXXXXX & $10847894-5$ & PAP -13 & BP CONDLOMA SE REAUIZA DCC & CONTROL EN $B$ MESES CON PAP & 10022005 \\
\hline $0 \times 0 \times \times \times 00 \times \times \times \times x$ & $15203059-K$ & WHE III TRATALAA & SE REALW $\triangle$ LEEP & CONTROL EN IN MES & 1. Hormans \\
\hline$x \times X \times 1 \times \times \times \times \times \times \times \times x$ & $5894739-0$ & PAPA & COLP' EPITELIOELANCO SE TOMA BP & OONTROL EN 3 SEMANAS & 04022005 \\
\hline$(6 \times \times \times \times \times \times \times O \times \times \times \times X$ & $1592427-3$ & CERYICOPATIA & COLP EXOSAINO & ALTA UPC & $16 \mathrm{OM} 2 \mathrm{xO}$ \\
\hline $00 \times \times x \times x \times x \times \times \times \times x$ & $8418383-0$ & CONELOWA TFATALO & COLF EXOC SANO & CONTFO ENE MESES CON FAF & 170282005 \\
\hline$\times \times \times \times \times \times \times \times \times \times \times \times \times \times x$ & 8756968.7 & NIE III TRETADA & COLP. SIKLLESION, SE TOMAPAD & CONTROL 6 MESES & 21022005 \\
\hline
\end{tabular}

Figura 3. Planilla de coordinación automática de cálculo de coberturas del Papanicolaou vigente, en mujeres de cada establecimiento le atención primaria de Servicio de Salud Valdivia. 


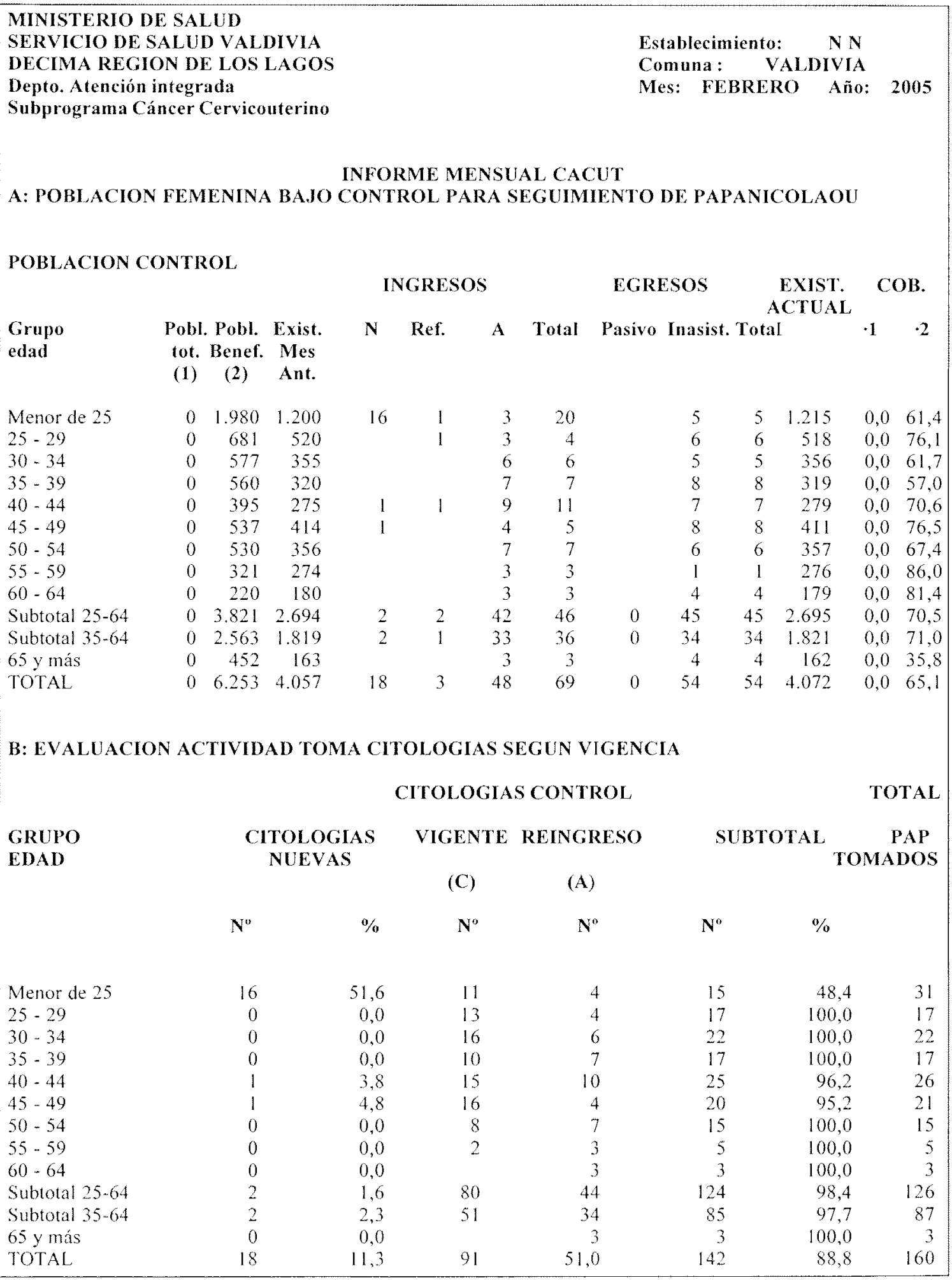

Figura 4. Planilla de coordinación de contra-referencia de usuarias evaluadas en Unidad de Patología Cervical, con destino a la atención primaria, vía e-mail, Servicio de Salud Valdivia. 
Estrategia $N^{0}$ 2. Incorporación de la comunidad al programa de detección precoz.

Se capacitó a 130 monitoras en Valdivia con talleres educativos sobre el Pap y la patología neoplásica, así como en técnicas de citación de sus iguales en las comunidades urbanas y rurales de las comunas de; Valdivia, La Unión, Rio Bueno, Panguipulli, Máfil, Lanco, Malalhue, Corral, Futrono, San José, Paillaco, Los Lagos, Lago Ranco, Coñaripe y Choshuenco, estas monitoras, además, tienen la importante labor de citar a mujeres inasistentes y nuevas para lo cual se organizan inter-sectorialmente en la comunidad y actúan como entidades autónomas, su idea incluso es generar recursos económicos propios que permitan financiar el traslado de pacientes de alta ruralidad y pobreza a Valdivia cuando necesiten ser evaluadas por un especialista.

Estrategia $\mathbf{N}^{\mathbf{0}}$ 3. Capacitación permanente e investigación operativa.

Se realiza capacitación permanente a matronas, médicos y monitoras de Pap sobre diagnóstico y tratamiento de la patología, uso del Cito-expert, evaluaciones e investigación operativa permanentes. Se reúne una vez al año a cada grupo de participantes en Valdivia, donde se presenta un diagnóstico epidemiológico y se acuerdan las tareas y capacitaciones a realizar durante el año siguiente (por ejemplo, calendario de pasantía de matronas nuevas por UPC, salidas del equipo coordinador a las localidades, programa de trabajo de monitoras y equipos locales tendientes al aumento de la cobertura, etc.)

Estrategia $N^{\circ} 4$ Implementación de un banco de proyectos de promoción.

Se creó un banco de proyectos de promoción concursable para equipos de salud y monitoras, cuyos fondos son los aportes que envía el Minsal para campañas sanitarias, en los cuales se les solicita como objetivo el aumento de la cobertura de Pap en sus comunidades. Así se ha logrado implementar infraestructura computacional en los establecimientos de atención primaria, formar a las monitoras, financiar campañas de difusión masiva, implementar Pap móviles y salas de toma de Pap, etc.

\section{Efecto del aumento de la cobertura del PAP vigente en la incidencia $y$ mortalidad por cáncer cervicouterino:}

El incremento en la cobertura que se obtuvo en el Servicio de Salud Metropolitano Norte, con la instalación y puesta en marcha del Citoexpert, fue de un $3 \%$ en el grupo de mujeres de 25 a 64 años y de un $8 \%$ en el grupo de mayor riesgo entre 35 a 64 años, durante el año 2003.

En el Servicio de Salud Valdivia, la cobertura del Pap vigente en las mujeres entre 25 a 64 años, ha evidenciado un aumento de un $12 \%$ desde la implementación de estas estrategias a partir del año 2000, obteniendo una cobertura de un 79\% en este grupo de edad. En el grupo de mayor riesgo, cuyas edades están entre los 35-64 años, la cobertura alcanzada es de un $77 \%$, con un incremento también de un $12 \%$ en los últimos años (Figura 5).

Respecto a las variaciones que ha sufrido la incidencia de la enfermedad, se evidencia un aumento en el número de casos nuevos de NIE III con tasas que van desde 29/100.000 mujeres (25 casos) en el año 1993, a 78/100.000 mujeres (102 casos) en el año 2003. La proporción de NIE III en relación al cáncer invasor ha mantenido un aumento constante y permanente en la última década: de 0,6 que existía en 1993 ha aumentado a 3,6 en el 2003, lo que significa tres casos de NIE HIl por cada caso de cáncer invasor. (Tabla 1).

La tasa de incidencia del cáncer invasor en el mismo periodo no ha variado, siendo de 35 $/ 100.000$ mujeres (41 casos) en el año 1993 y $35 / 100.000$ mujeres ( 45 casos) en el año 2003, pero sí la detección ha favorecido las etapas tempranas, donde la frecuencia de estadios de invasión precoz (I y II) es cada vez mayor respecto a los estadios de invasión avanzada (IIf y IV), lo que epidemiológicamente es esperable en un programa en que se está logrando éxito. (Figura 6).

La disminución de la tasa de mortalidad en el Servicio de Salud Valdivia, claramente expuesta en la Tabla 1 y Figura 5 , y que para el último año de nuestro análisis es de 4,6 por 100.000 mujeres mayores de 15 años, nos estimula porque es el reflejo del trabajo que está realizando todo un equipo que con 

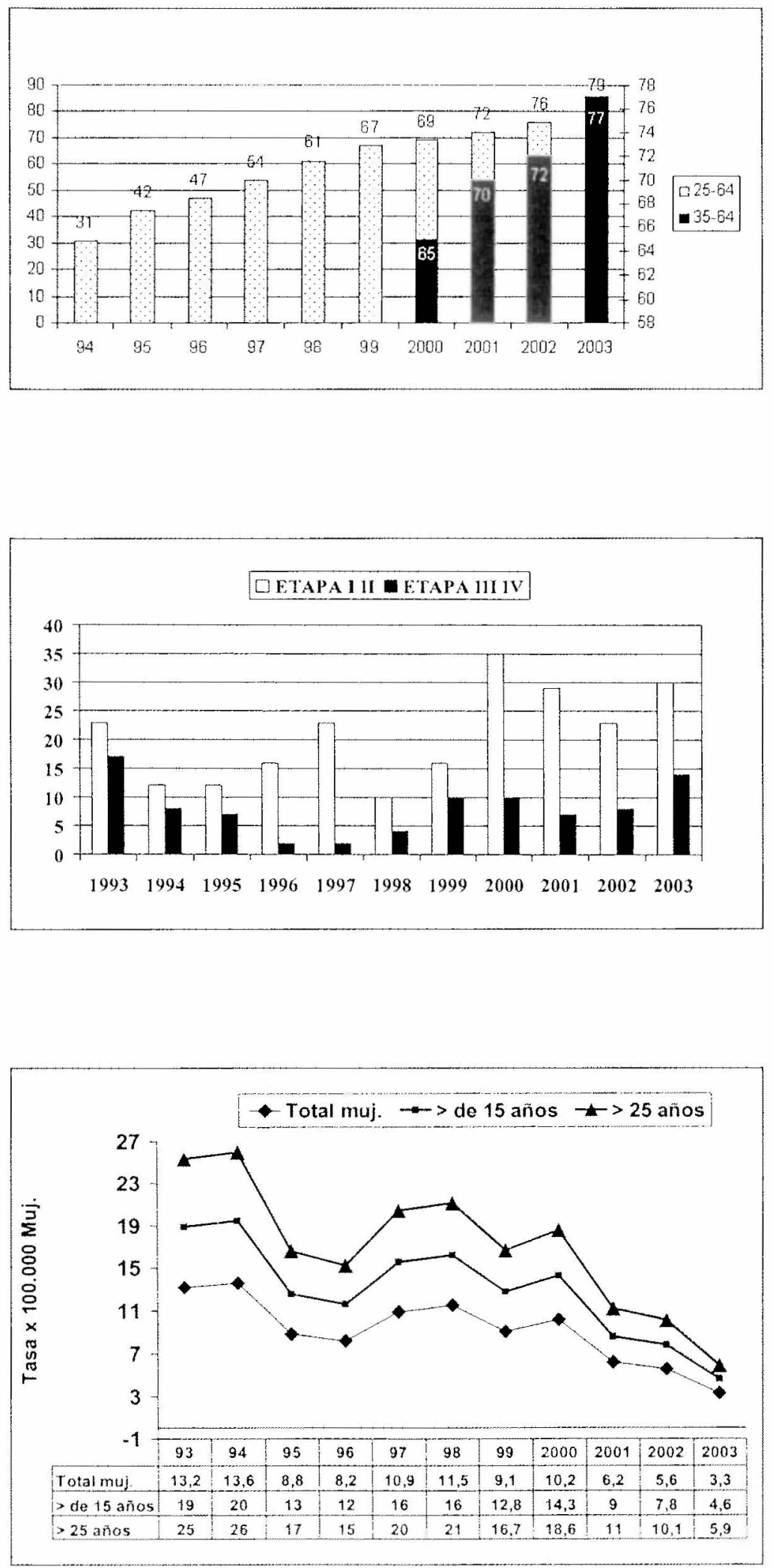

Figura 5. Distribución porcentual de la cobertura del Papanicolaou vigente en mujeres beneficiarias del Servicio de Salud Valdivia, periodo 1994 al 2003. Fuente: Servicio de Salud Valdivia.

Figura 6. Variación en la distribución por etapas clínicas del éncer cervicouterino invasor Servicio de Salud Valdivia, periodo 1993 al 2003. Fuente: diagnóstico histológico Servicio Anatomia Patológica Hospital Clínico Regional Valdivia

Figura 7. Tasa de mortalidad por cáncer cervicouterino por 100.000 mujeres. Tasas total y específica por edad. Periodo 1994 al 2003, Servicio de Salud Valdivia. Fuente: certificados de defunción y registros Unidad de Patologia Cervical, Servicio de Salud Valdivia. 
Tabla 1. Número y proporción de cáncer in situ (NIE III) y cáncer invasor por año, Servicio de Salud Valdivia

\begin{tabular}{cccc}
\hline Año & $\begin{array}{c}\text { Cáncer in situ } \\
\mathrm{n}\end{array}$ & $\begin{array}{c}\text { Cáncer invasor } \\
\mathrm{n}\end{array}$ & Proporción \\
\hline 1993 & 25 & 41 & 0,6 \\
1994 & 49 & 20 & 2,45 \\
1995 & 38 & 20 & 1,9 \\
1996 & 48 & 18 & 2,6 \\
1997 & 48 & 25 & 1,9 \\
1998 & 46 & 14 & 3,3 \\
1999 & 36 & 26 & 1,4 \\
2000 & 72 & 45 & 1.6 \\
2001 & 100 & 36 & 2,8 \\
2002 & 95 & 31 & 3,1 \\
2003 & 102 & 45 & 2,3 \\
\hline
\end{tabular}

Fuente: Cito-expert, Anatomia Patológica Hospital Clínico Regional Valdivia.

motivación y esfuerzo, está demostrando que es posible modificar favorablemente nuestros indicadores sanitarios, a pesar de la habitual insuficiencia de recursos (Figura 7).

\section{DISCUSIÓN}

El aumento en las coberturas del Pap va asociado a un conjunto de estrategias que dependen esencialmente de la motivación y aceptabilidad de esta acción, como un desafio de los equipos de salud de los establecimientos de atención primaria y la coordinación del programa a cargo de las matronas de cada localidad, así como del acceso físico y del vencer barreras sicológicas (miedo, pudor, errores de información y creencias), que impiden a muchas mujeres acercarse a realizar el Pap ${ }^{7}$.

La historia natural de la patología neoplásica cervical que se detecta a través del Pap, implica la progresión gradual por etapas intraepiteliales preinvasoras como son las Neoplasia intraepitelial NIE I, II y lll o carcinonta in situ 8,9 por lo tanto, lo más importante es la detección precoz. En nuestro país, y por razones de costo, el programa organizado de pesquisa debería ser la primera alternativa dada la eficiencia y eficacia demostrada en los países desarrollados, sin embargo, para lograrlo se debe alcanzar y mantener, por lo menos, una cobertura de un
$80 \%$ de Pap vigente a largo plazo en la población expuesta al riesgo $\mathrm{o}^{2,3}$.

La implementación de estrategias comunicacionales, de salud pública y gestión entre las UPC y la atención primaria, así como el uso de la informática y fomento de la participación comunitaria, la capacitación y la investigación operativa, permitieron aumentar la cobertura del Pap vigente y el diagnóstico de lesiones preinvasoras logrando un descenso en la mortalidad.

\section{REFERENCIAS}

1.- FRANCO E L, FRANCO E D, FERENCZY A. Cervical cancer: epidemiology, prevention and the role of human papillomavirus infection. CMAJ 2001; $164(7)$.

2.- QUINN M, BABB P, JONES J, ALLEN E. Effect of screening on incidence of and mortality from cervical cancer in England: evaluation based on routinely collected statistic. BMJ 1999; $318: 904$.

3.- MAC GREGOR J E, CAMPBELLO M K, MANN E M, SWANSON K Y: Sereening for cervical intraepithelial neoplasia North East Scotland shows fall in incidence and mortality from invasive cancer with concomitant rise in preinvasive disease. BMJ 1994; 308: 1407-11.

4.- SUÁREZ E, PRIETO M, ROJAS 11, FERNÁNDEZ B, PRADO R, OLFOS P. Programa Nacional de cáncer cervicouterino. Rev Chil Obstet Ginecol $2001 ; 66(6)$ : 480-91.

5.- SERRA 1, SERRA L, CASTRO R, GARCÍA V, SERRA J, DECINTI E, CARCAMO S. Cáncer cervicouterino, un desafio sin resolver. Rev Chil Obstet Ginecol 1997; 62(2): 75-85.

6.- Instituto Nacional de Estadísticas, Ministerio de Economia: Chile, estimaciones y proyecciones de población por sexo y edad. Total pais: 1950-2050.

7.- VALENZUELA M T, MIRANDA A. ¿Por qué No me hago el Papanicolau? Barreras sicológicas de mujeres de sectores populares de Santiago de Chile. Rev Chil Salud Pública 2001; 5 (2-3): 75-80.

8.- RICHART R M. Natural history of cervical intraepithelial neoplasia. Clin Obstet Gynecol 1967; 10: 748-84.

9.- RICHART R M. Natural history of cervical intraepithelial neoplasia. Obstet Gynecol 1990; 75: $\$ 31-3 c$.

Agradecimientos: A todas y cada una de las matronas que trabajan en la atención primaria que permitienon desarrollar y ejecutar este proyecto. Al Dr. Iván Serra, docente Escuela de Salud Pública Universidad de Chile y Sra. Mireya Faria C. Bibliotecaria, biblioteca Medicina Univ. Austral de Chile, por apoyo prestado y al colegio de Matronas de Chile, con especial éntasis al Consejo Regional Valdivia. 